\title{
Overexpression of HS6ST2 is associated with poor prognosis in patients with gastric cancer
}

\author{
YI JIN $^{1^{*}}$, JUN HE ${ }^{2 *}$, JING DU $^{3}$, RU-XUAN ZHANG ${ }^{4}$, HAI-BO YAO $^{2}$ and QIN-SHU SHAO ${ }^{2}$ \\ ${ }^{1}$ Department of General Surgery, Children's Hospital, School of Medicine, Zhejiang University, Hangzhou, Zhejiang 310052; \\ Departments of ${ }^{2}$ Gastroenterology and Pancreatic Surgery and ${ }^{3}$ Gastroenterology, Zhejiang Provincial People's Hospital, \\ Hangzhou, Zhejiang 310014; ${ }^{4}$ Zhejiang Academy of Medical Sciences, Hangzhou, Zhejiang 310013, P.R. China
}

Received May 23, 2016; Accepted June 15, 2017

DOI: $10.3892 / \mathrm{ol} .2017 .6944$

\begin{abstract}
The purpose of the present study was to investigate the clinical significance of the expression of heparan sulfate 6-O-sulfotransferase 2 (HS6ST2) in gastric cancer (GC). The Affymetrix GeneChip ${ }^{\circledR}$ Human Genome U133 Plus 2.0 Array (Affymetrix; Thermo Fisher Scientific, Inc., Waltham, MA, USA) was used to identify differentially expressed genes in GC tissues vs. adjacent non-tumor gastric tissues. Candidate genes were further verified by reverse transcription quantitative polymerase chain reaction (RT-qPCR) and immunohistochemistry (IHC). In addition, an independent dataset was obtained from the Gene Expression Omnibus, and a survival analysis was performed. Microarray analysis demonstrated that HS6ST2 was upregulated (>12-fold) in GC tissues compared with that in adjacent non-tumor tissues. RT-qPCR and IHC analysis of HS6ST2 in GC tissues and adjacent non-tumor tissues confirmed the microarray data. Furthermore, a positive association was demonstrated between HS6ST2 overexpression with the depth of tumor invasion, distant metastasis, and tumor-node metastasis stage. Survival analysis revealed an association between patients with increased expression of HS6ST2 and a poor prognosis of gastric cancer. Cox regression analysis indicated that the expression of HS6ST2 was an independent negative prognostic factor for GC. The expression of HS6ST2 in GC was significantly associated with specific clinicopathological parameters and prognosis of disease, thus we propose that HS6ST2 may represent a novel biomarker for GC.
\end{abstract}

Correspondence to: Professor Qin-Shu Shao, Department of Gastroenterology and Pancreatic Surgery, Zhejiang Provincial People's Hospital, 158 Shangtang Road, Hangzhou, Zhejiang 310014, P.R. China.

E-mail: zhumf@hzswdx.gov.cn

${ }^{*}$ Contributed equally

Key words: heparin sulfate 6-O-sulfotransferase 2, microarray, gastric cancer, prognosis, biomarker

\section{Introduction}

Gastric cancer (GC) is the fifth most frequently diagnosed cancer, and a leading cause of cancer-associated mortality, with $\sim 1,000,000$ new cases reported and resulting in $\sim 723,000$ mortalities globally in 2012 (1). In China, GC is the third most common type of cancer and a leading cause of cancer-related mortality; with 420,000 new cases and 290,000 mortalities reported in 2011 (2). Despite progress being made in the diagnosis and treatment of GC, the long-term survival rate remains relatively low, with $70-75 \%$ of patients with GC ultimately succumbing to the disease (3). As a consequence, there is an urgent requirement to identify novel prognostic factors in patients with GC (4). Furthermore, identifying molecular subgroups associated with GC may lead to identifying patients who may potentially benefit from targeted drug therapies (5). The pathogenesis of gastric carcinogenesis remains incompletely characterized and there are only a limited number of reliable molecular biomarkers, which are currently targeted in clinical practice. Therefore, the challenge remains to improve our understanding of the molecular mechanisms underlying $\mathrm{GC}$, in order to identify biomarkers for early diagnosis, prognosis prediction and potential therapeutic targets.

The heparan sulfate 6-O-sulfotransferase (HS6ST) family comprises three isoforms (HS6ST1, 2, and 3), whose major function consists of attaching a sulfate to heparan sulfate proteoglycans (HSPGs) (6). HS6ST2, a member of the HS6ST family, has an alternatively spliced form named HS6ST-2S with 40 amino acids deleted. Despite the differences in sequences, the two enzymes catalyze the transfer of sulfate groups from adenosine 3'-phosphate, 5'-phosphosulphate (PAPS) to the 6-O position of the glucosamine residues in HSPGs (7). By way of this mechanism, HSPGs subsequently participate in diverse biological functions including blood clotting, cell recognition, adhesion, proliferation, and differentiation by interactions with diverse cytokines $(8,9)$. Previous studies have revealed that HS6ST2 is associated with the progression of malignant tumors, and is upregulated in various tumor types including thyroid $(10,11)$, colorectal (12), pancreatic (13), ovarian (14), breast cancer (15), and chondrosarcomas (16). However, to the best of our knowledge, the involvement of HS6ST2 in GC has not yet been elucidated. In the present study, we evaluated the expression of HS6ST2 in GC and non-tumor tissues, and 
evaluated the association with clinicopathological features and prognostic values, to determine whether HS6ST2 represents a novel biomarker for patients with GC.

\section{Materials and methods}

Human tissue specimens. A total of 46 paired fresh tumor and adjacent non-tumor tissue samples were collected from patients who underwent curative surgery for gastric cancer at Zhejiang Provincial People's Hospital, (Zhejiang, China), between January 2011 and December 2012 and January 2006 and December 2008. A total of 110 fresh tissues were formalin-fixed, paraffin-embedded or immediately snap frozen in liquid nitrogen $\left(-196^{\circ} \mathrm{C}\right)$ following resection and stored at $-80^{\circ} \mathrm{C}$ until analysis. A total of 10 paired samples were profiled on the GeneChip Human Genome U133 Plus 2.0 Array platform (Affymetrix; Thermo Fisher Scientific, Inc.) and 36 paired samples were used for reverse transcription quantitative polymerase chain reaction analysis (RT-qPCR). Patient ages ranged from 30-81 years (median age, 59 years), of which 80 were males and 30 were females. Tumor location, tumor size, differentiation status, invasion depth, lymph node metastasis, distant metastasis and tumor-node metastasis (TNM) stage were also recorded. The pathological diagnosis was confirmed independently by two pathologists, according to the 7th edition of the American Joint Committee on Cancer staging manual (17). The last follow-up was December 2015. In addition, 60 non-tumor gastric tissue specimens were acquired by endoscopy from patients without tumors and used as controls.

The present study was approved by the Ethics Committee of Zhejiang Provincial People's Hospital. Written informed consent was obtained from all patients enrolled in the present study, for the use of all resultant specimens. None of the patients enrolled in the present study had received chemoradiotherapy treatment prior to surgery.

Microarray analysis. Total RNA was extracted using TRIzol ${ }^{\circledR}$ reagent (Invitrogen; Thermo Fisher Scientific, Inc.) and examined using an Agilent Bioanalyzer 2100 (Agilent Technologies, Inc., Santa Clara, CA, USA) according to the manufacturer's protocol. Total RNA was amplified, labeled, and purified to obtain biotin labeled cRNA using a GeneChip ${ }^{\circledR}$ 3'IVT Express kit (Affymetrix; Thermo Fisher Scientific Inc.) Array hybridization and washes were performed using GeneChip ${ }^{\circledR}$ Hybridization, Wash and Stain kit (Affymetrix; Thermo Fisher Scientific Inc.), and a Hybridization Oven 645 and a Fluidics Station 450 (Affymetrix; Inc). Slides were scanned using GeneChip ${ }^{\circledast}$ Scanner 3000 (Affymetrix; Thermo Fisher Scientific Inc.) and Command Console Software 3.1 (Affymetrix; Thermo Fisher Scientific Inc.) with default settings. Raw data were normalized by MAS 5.0 algorithm in Gene Spring Software 11.0 (Agilent Technologies, Inc.). Differentially expressed genes in GC compared with adjacent non-tumor tissues were identified through SAM (significance analysis of microarray). Genes were regarded as differentially expressed when the $\mathrm{N}$ (normal) vs. $\mathrm{T}$ (tumor) signal log ratio values were $\leq 0.5$ or $\geq 2$.

Reverse transcription quantitative polymerase chain reaction (RT-qPCR) analysis. The expression of HS6ST2 mRNA was evaluated by RT-qPCR analysis. Total RNA was isolated using TRIzol ${ }^{\circledR}$ reagent (Invitrogen; Thermo Fisher Scientific, Inc.) and reverse-transcribed to cDNA using a PrimeScript ${ }^{\mathrm{TM}}$ RT Reagent kit (Takara Biotechnology Co., Ltd., Dalian, China), according to the manufacturer's protocol. Glyceraldehyde-3-phosphate dehydrogenase $(G A P D H)$ was selected as an internal control. qPCR was performed in a $20 \mu 1$ total reaction volume using SYBR $^{\circledR}$ Premix Ex Taq ${ }^{T M}$ II kit (Takara Biotechnology Co., Ltd.) on the Mx3000P qPCR System (Stratagene; Agilent Technologies, Inc.). The primer sequences were as follows: HS6ST2 forward, 5'-GAAGCAGAACTCAGGCAAGG-3' and reverse, 5'-CCAATGAAGGAAGCAGGATGT-3'; GAPDH forward, 5'-TGAAGGTCGGAGTCAACGG-3' and reverse, 5'-CTGGAAGATGGTGATGGGATT-3'. The PCR reaction was run as follows: $95^{\circ} \mathrm{C}$ for $4 \mathrm{~min}$ as an initial denaturation step, amplification for 40 cycles with denaturation at $95^{\circ} \mathrm{C}$ for $10 \mathrm{sec}$, annealing at $57^{\circ} \mathrm{C}$ for $30 \mathrm{sec}$, and extension at $72^{\circ} \mathrm{C}$ for $30 \mathrm{sec}$. Melting curve analysis was performed at the termination of the PCR cycle. Each qPCR was performed in triplicate, from which the mean value was calculated. The expression level of HS6ST2 mRNA was expressed as $2^{-\triangle \Delta C q}$ in which $\mathrm{DCq}=\mathrm{Cq}$ (HS6ST2)-Cq (GAPDH) (18).

Immunohistochemistry (IHC) analysis. Paraffin-embedded tissues $(\mathrm{n}=110)$, which were formalin fixed in $10 \%(\mathrm{v} / \mathrm{v})$ formalin for $24 \mathrm{~h}$ at room temperature and cut into $4 \mu \mathrm{m}$ sections for IHC. Sections were deparaffinized and rehydrated through a descending series of alcohols. Tissue sections were placed in a $10 \mathrm{mM}$ sodium citrate buffer $(\mathrm{pH}, 6.0)\left(120^{\circ} \mathrm{C}\right.$ for $3 \mathrm{~min}$ ) to perform antigen retrieval and washed by PBS. An SP-9000 Detection kit (OriGene Technologies, Inc., Beijing, China) was used for performing IHC. Endogenous peroxidase activity was blocked by $3 \%(\mathrm{v} / \mathrm{v})$ hydrogen peroxide for $15 \mathrm{~min}$ at room temperature. Non-specific binding was blocked by incubation with $10 \%$ (v/v) goat serum (OriGene Technologies, Inc., Beijing, China) or $20 \mathrm{~min}$ at room temperature. The sections were incubated overnight at $4^{\circ} \mathrm{C}$ with a primary antibody against HS6ST2 (1:200; cat. no. ab122220; Abcam, Cambridge, UK). The sections were then incubated with a biotin-labeled secondary antibody (ready-to-use kit; cat. no. SP9000; goat anti-mouse IgG; OriGene Technologies, Inc., Beijing, China) for $15 \mathrm{~min}$ at room temperature and incubated with horseradish peroxidase-labeled streptavidin (OriGene Technologies, Inc.) for $20 \mathrm{~min}$ at room temperature. Slides were stained with 3,3'-diaminobenzidine (DAB; OriGene Technologies, Inc.) for $5 \mathrm{~min}$ at room temperature and counterstained with hematoxylin. For the negative control, immunohistochemical staining was performed by omitting the primary anti-HS6ST2 antibody.

All slides were independently examined by two pathologists (Department of Pathology, Zhejiang Provincial People's Hospital Zhejiang, China) blinded to the individual patient data, using an optical microscope (Nikon Corporation, Tokyo, Japan) to examine five random fields of view at magnification, x200 and x400. The immunoreactivity of HS6ST2 was evaluated according to a combined scoring system based on the staining intensity and percentage of positive stained cells. Intensity scores from 0 to 3 were defined as follows: 0 , no staining; $1+$, weak staining; $2+$, moderate staining and $3+$, strong staining. The extent of staining was scored from 
Table I. Selected genes significantly upregulated in gastric cancer.

\begin{tabular}{|c|c|c|c|c|c|c|}
\hline Probe set ID & $\begin{array}{l}\text { False discovery } \\
\text { rate (FDR) }\end{array}$ & $\begin{array}{l}\text { Fold-change } \\
\text { (T vs. N) }\end{array}$ & $\begin{array}{c}\text { Gene } \\
\text { symbol }\end{array}$ & $\begin{array}{c}\text { Entrez } \\
\text { Gene ID }\end{array}$ & $\begin{array}{c}\text { Chromosomal } \\
\text { location }\end{array}$ & $\begin{array}{l}\text { UniGene } \\
\text { ID }\end{array}$ \\
\hline 227140_at & 0.000326 & 27.848961 & $I N H B A$ & 3624 & chr7p15-p13 & Hs. .583348 \\
\hline 203820_s_at & 0.001551 & 16.163251 & $I G F 2 B P 3$ & 10643 & chr7p11 & Hs.700696 \\
\hline 204051_s_at & 0.001501 & 15.832003 & SFRP4 & 6424 & chr7p14.1 & Hs.658169 \\
\hline 212353_at & 0.000653 & 13.410469 & SULF1 & 23213 & chr8q13.1 & Hs. 409602 \\
\hline 230030_at & 0.009619 & 12.400589 & HS6ST2 & 90161 & $\operatorname{chrXq26.2}$ & Hs.385956 \\
\hline 226237_at & 0.000653 & 11.848787 & COL8A1 & 1295 & $\operatorname{chr} 3 q 12.3$ & Hs. 654548 \\
\hline 209875_s_at & 0.001303 & 10.858137 & $S P P 1$ & 6696 & chr4q22.1 & Hs.313 \\
\hline 223475_at & 0.002232 & 9.8014127 & CRISPLDI & 83690 & chr8q21.11 & Hs. 436542 \\
\hline 219087_at & 0.001643 & 8.9984428 & $A S P N$ & 54829 & $\operatorname{chr9q22}$ & Hs .435655 \\
\hline 213905_x_at & 0.002647 & 8.8140516 & $B G N$ & 633 & $\operatorname{chrXq} 28$ & Hs.821 \\
\hline 225681_at & 0.000595 & 8.4565955 & CTHRC1 & 115908 & chr8q22.3 & Hs. 405614 \\
\hline 202363_at & 0.003312 & 8.0317287 & SPOCK1 & 6695 & chr5q31 & Hs.596136 \\
\hline 225664_at & 0.001047 & 7.6351305 & COL12A1 & 1303 & chr6q12-q13 & Hs.101302 \\
\hline 227566_at & 0.000806 & 7.4371010 & NTM & 50863 & chr11q25 & Hs.504352 \\
\hline
\end{tabular}

$\mathrm{T}$, tumor tissue; $\mathrm{N}$, adjacent non-tumor tissue; FDR $<0.05$ was considered to represent a statistically significant difference.

0 to 4 as follows: $0,<5 \%$ positive cells; $1+, 5-25 \%$ positive cells; $2+, 26-50 \%$ positive cells; $3+, 51-75 \%$ positive cells; and $4+, 76-100 \%$ positive cells. Individual patient scores were obtained by multiplying the intensity and extent score. Scores from 0 to 3 were regarded as negative staining, while scores from 4 to 12 were deemed positive.

Gene expression omnibus dataset and survival analysis. A gene expression dataset composed of 876 patients with gastric cancer was obtained as previously described (19). In brief, the key words 'gastric', 'cancer', 'GPL570' and 'GPL96' were searched for in the GEO dataset (http://www.ncbi.nlm. nih.gov/geo/) (20). Publications containing only raw gene expression files, survival information, and a minimum of 30 patients were used for subsequent analyses. Raw. CEL files were MAS 5.0 normalized using R (http://www.r-project. org). A second scaling normalization was performed to reduce batch effects (21). In the present study, the expression level of HS6ST2 was evaluated in $629 \mathrm{GC}$ samples from the merged dataset. All percentiles between lower and upper quartiles of HS6ST2 were computed and the best performing threshold was used as cutoff (cutoff =31) (22). GraphPad Prism ${ }^{\circledR}$ version 5.0, GraphPad Software Inc., La Jolla, CA, USA) was employed for Kaplan-Meier analysis.

Statistical analysis. Statistical analysis was performed using SPSS (version 19.0; IBM Corp., Armork, NY, USA). The mRNA expression of HS6ST2 in paired adjacent non-tumor and tumor samples was evaluated using the Wilcoxon signed-rank test. The association between the expression of HS6ST2 protein and clinicopathological data was performed using a $\chi^{2}$ test. The survival analyses were evaluated by Kaplan-Meier survival curves and the differences between the two groups were compared by a log-rank test. The survival data was also evaluated by univariate and multivariate Cox regression analysis. $\mathrm{P}<0.05$ was considered to indicate a statistically significant difference.

\section{Results}

Evaluation of HS6ST2 mRNA expression by microarray analysis. In the present study, differentially expressed genes between 10 pairs of adjacent non-tumor and gastric cancer tissues were analyzed by microarray, and the raw data was uploaded to GEO (accession no. GSE79973). The results from the present study indicated that the expression of HS6ST2 was significantly upregulated in GC tissues compared with adjacent non-tumor tissues ( $>12$-fold; false discovery rate $<0.01$; Table I). As a result, HS6ST2 was selected as a potential biomarker, and its involvement in GC was further investigated.

HS6ST2 mRNA expression in GC and adjacent non-tumor tissues. In the present study, the microarray data was further validated by RT-qPCR analysis of the expression of HS6ST2 mRNA in 36 paired GC and adjacent non-tumor specimens. The expression of HS6ST2 mRNA was significantly increased in tumor tissue compared with that of adjacent non-tumor tissue in $29(80.6 \%)$ cases. The mean expression level of HS6ST2 mRNA in GC was significantly higher than that observed in non-tumor tissues $(\mathrm{P}<0.001$; Fig. 1A). The relative HS6ST2 mRNA expression level of each specimen was presented in Fig. $1 \mathrm{~B}$.

HS6ST2 protein levels in GC and non-tumor tissues. In the present study, HS6ST2 protein levels were examined in 110 GC tissues and 60 non-tumor gastric tissues by IHC. Positive immunostaining for HS6ST2 protein was high in GC (70.9\%; 78/110) compared with that observed in non-tumor gastric mucosa $(18.3 \%, 11 / 60 ; \mathrm{P}<0.001)$. HS6ST2 staining was detected by a brown stain in the cytoplasm of cells (Fig. 2). 
Table II. Associations between HS6ST2 expression and clinicopathological data.

HS6ST2

expression

Parameter Cases (n) Positive Negative P-value

\begin{tabular}{llrr}
\hline Sex & & & \\
Male & 80 & 56 & 24 \\
Female & 30 & 22 & 8 \\
$\begin{array}{l}\text { Age, years } \\
\geq 60\end{array}$ & & & \\
$<60$ & 54 & 40 & 14 \\
$\quad$ Tumor size, mm & & 38 & 18 \\
$\geq 50$ & 62 & 45 & 17 \\
$<50$ & 48 & 33 & 15
\end{tabular}

Tumor location

$\begin{array}{lrrr}\text { Fundus } & 26 & 20 & 6 \\ \text { Body } & 38 & 28 & 10 \\ \text { Antrum } & 46 & 30 & 16\end{array}$

Differentiation

Well or moderate

Poor

$44 \quad 32$

$66 \quad 46$

12

20

Local invasion

$\mathrm{T}_{1}-\mathrm{T}_{2}$

$26 \quad 14$

12

84

64

20

Node metastasis

Yes

$81 \quad 58$

2920

23

No

0.732

8

14

18

0.661

Distant metastasis

$\begin{array}{lrrr}\text { Yes } & 24 & 22 & 2 \\ \text { No } & 86 & 56 & 30\end{array}$

TNM stage

\begin{tabular}{lrrr} 
I & 13 & 7 & 6 \\
II & 22 & 13 & 9 \\
III & 51 & 36 & 15 \\
IV & 24 & 22 & 2 \\
\hline
\end{tabular}

${ }^{\mathrm{a} P}<0.05$, based on a $\chi^{2}$ test. HS6ST2, Heparan sulfate 6-O-sulfotransferase 2; TNM, tumor-node metastasis.

Associations between HS6ST2 expression and clinicopathological features. The analysis of the association between HS6ST2 protein expression and clinicopathological parameters, within the cohort were then evaluated using a $\chi^{2}$ test. A positive association was observed between the expression of HS6ST2 protein with invasion depth $(\mathrm{P}=0.028)$, distant metastasis $(\mathrm{P}=0.011)$, and tumor-node metastasis stage $(\mathrm{P}=0.039)$. However, associations were not observed between HS6ST2 expression and patient sex $(\mathrm{P}=0.732)$, age $(\mathrm{P}=0.473)$, tumor size $(\mathrm{P}=0.661)$, tumor location $(\mathrm{P}=0.517)$, lymph node metastasis $(\mathrm{P}=0.788)$ or the degree of pathological differentiation ( $\mathrm{P}=0.732$; Table II).
A

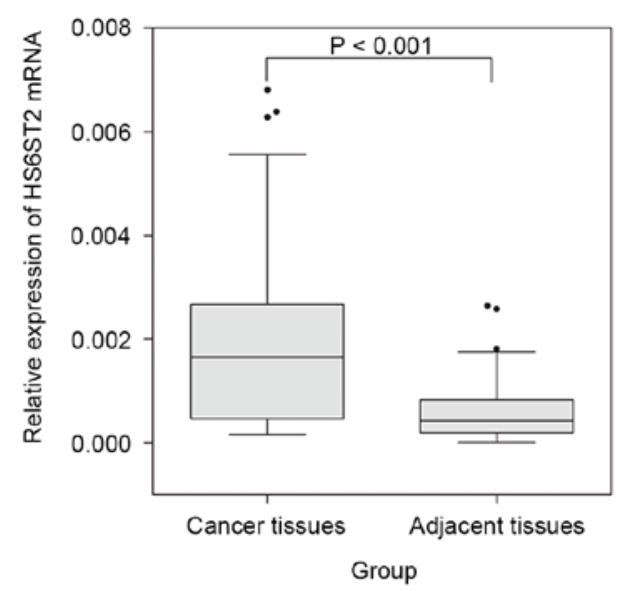

B

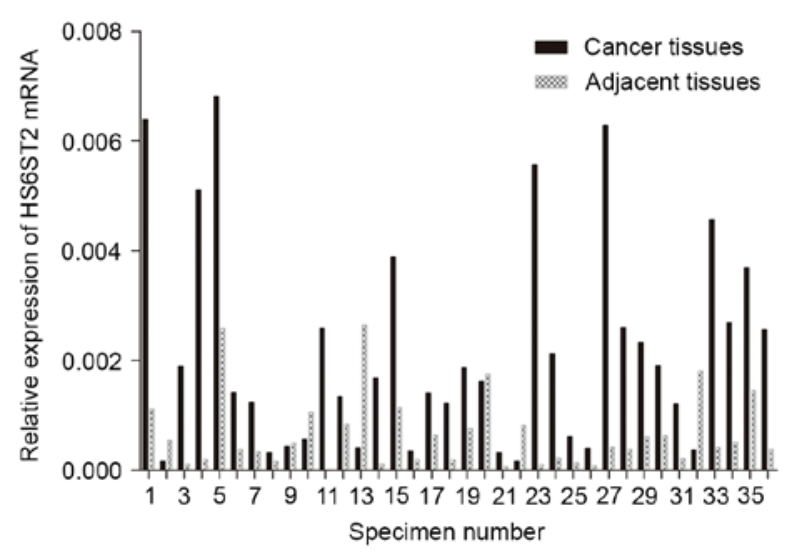

Figure 1. Reverse transcription-quantitative polymerase chain reaction analysis of HS6ST2 in gastric cancer and adjacent non-tumor tissues. (A) Relative expression levels of HS6ST2 mRNA in gastric cancer tissues was significantly higher than that of adjacent tissues. (B) Relative HS6ST2 mRNA expression levels in 36 paired specimens. HS6ST2, heparan sulfate 6-O-sulfotransferase 2.

Overall survival analysis. Overall survival analysis based on IHC and evaluated by Kaplan-Meier curves, revealed that the 5-year survival rate of the HS6ST2-positive (28\%) group was significantly reduced compared with the HS6ST2-negative group (64.7\%; Fig. 3A). Cox regression analysis indicated that the independent negative prognostic factors for GC were HS6ST2 protein expression $(\mathrm{P}=0.038)$ and local invasion $(\mathrm{P}=0.031$; Table III). These results were validated using a non-overlapping cohort of 629 patients. In the validation cohort, patients with low expression of HS6ST2 demonstrated increased overall survival, compared with the HS6ST2-high group $(\mathrm{P}=0.0002)$. Furthermore, the 5-year survival rate in HS6ST2-high group was 41.9\% compared with $63.1 \%$ in HS6ST2-low group (Fig. 3B).

\section{Discussion}

The treatment of gastric cancer currently involves combinatorial therapies, including surgery, chemotherapy and radiotherapy; however, long-term survival rates remain relatively low $(3,23)$. TNM classification based on the clinicopathological characteristics of tumors; including local invasive depth, lymph node involvement, and distant metastasis is the current gold standard for prognosis prediction and available 
Table III. Multivariate Cox regression survival analysis of clinicopathological data and HS6ST2 expression in patients with gastric cancer.

\begin{tabular}{lcccc}
\hline Variable & Cases (n) & Hazard ratio & 95\% confidence interval & P-value \\
\hline Tumor location, fundus/body/antrum & $26 / 38 / 46$ & 0.748 & $0.551-1.104$ & 0.062 \\
Local invasion, T3-T4/T1-T2 & $84 / 26$ & 2.938 & $1.106-7.805$ & $0.031^{\text {a }}$ \\
Node metastasis, yes/no & $81 / 29$ & 1.201 & $0.507-2.843$ & 0.677 \\
Distant metastasis, yes/no & $24 / 86$ & 2.215 & $0.764-6.422$ & 0.143 \\
TNM stage, IV/III/II/I & $24 / 51 / 22 / 13$ & 1.452 & $0.671-3.139$ & 0.344 \\
HS6ST2 expression, positive/negative & $78 / 32$ & 2.042 & $1.040-4.012$ & $0.038^{\mathrm{a}}$ \\
\hline
\end{tabular}

${ }^{\mathrm{a}} \mathrm{P}<0.05$. TNM, tumor-node metastasis; HS6ST2, heparan sulfate 6-O-sulfotransferase 2.

A

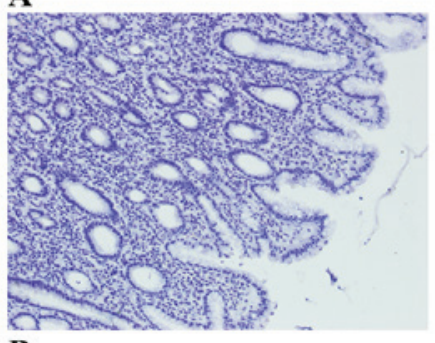

B

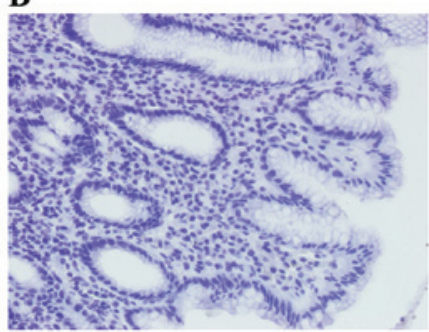

C

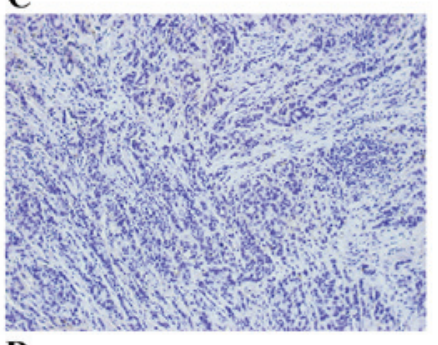

D

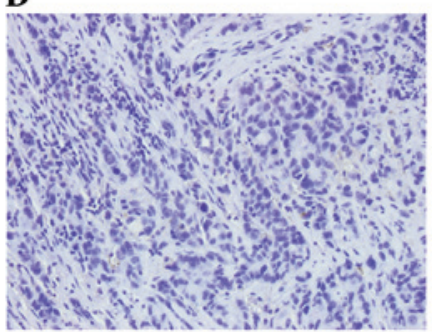

$\mathbf{E}$

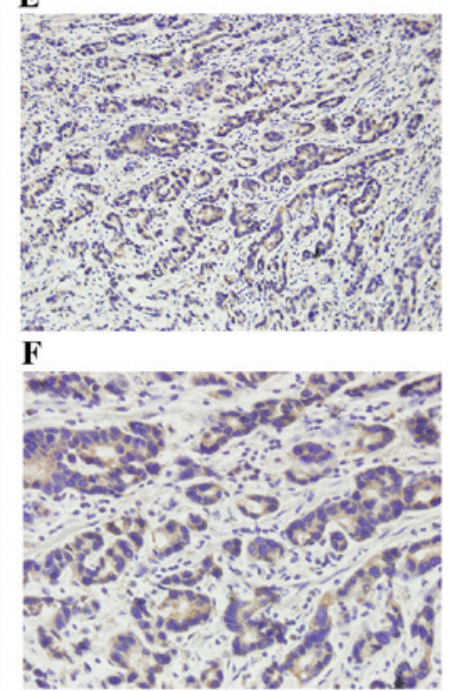

Figure 2. Immunohistochemical analysis of HS6ST2 in gastric cancer and non-tumor tissues. Negative staining of HS6ST2 in non-tumor gastric tissue at magnification, (A) x200 and (B) x400. Negative staining of HS6ST2 in gastric cancer tissue at magnification, (C) x200 and (D) x400. Positive staining of HS6ST2 in GC tissue at magnification, (E) x200 and (F) x400. HS6ST2, heparan sulfate 6-O-sulfotransferase 2; GC, gastric cancer.
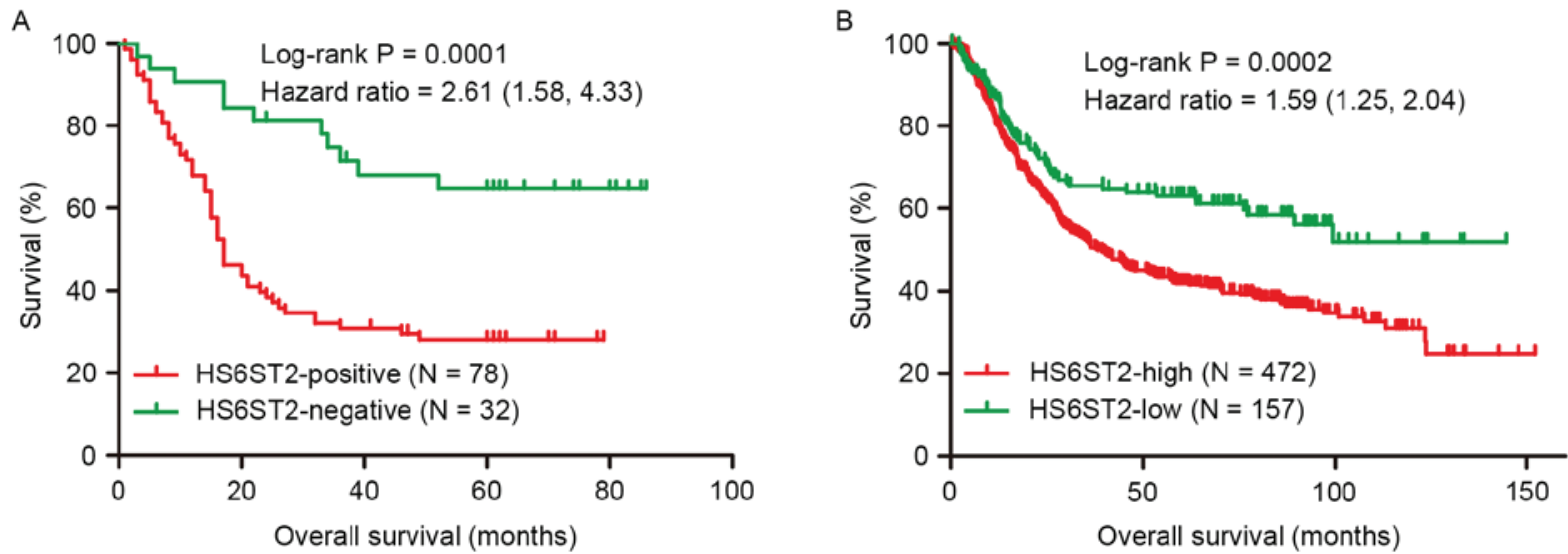

Figure 3. Kaplan-Meier survival curves for patients with differing HS6ST2- expression. (A) Kaplan-Meier survival analysis based on immunohistochemistry indicated that the prognosis of the HS6ST2-positive group was lower than that of HS6ST2-negative group. (B) Kaplan-Meier survival analysis based on a merged dataset indicated that the prognosis of HS6ST2-high group was lower than that of HS6ST2-low group. HS6ST2, heparan sulfate 6-O-sulfotransferase 2.

treatment options for GC $(24,25)$. However, even when symptoms of GC are detected at the early stages, patients are still susceptible to a reduced life span (25).
In previous decades, molecular profiling has redefined our understanding of multiple types of human cancer. Molecular biomarkers are not only useful for diagnosis but may represent 
potential targets with prognostic significance in multiple types of cancer (26). However, there are a limited number of biomarkers identified, which are specific to GC. The application of microarray technology has proved beneficial in the investigation of the differential gene expression profile of $\mathrm{GC}$ and the identification of genes specifically expressed in GC (27). Thus, the microarray-based approach in the present study was used to screen for genes aberrantly expressed in GC, and to select novel molecular biomarkers, which may prove to have prognostic significance in GC. In the present study, microarray was used to analyze differentially-expressed genes in 10 pairs of adjacent non-tumor and gastric cancer tissues, and the result showed that the expression of HS6ST2 was significantly upregulated in GC tissues compared with adjacent non-tumor tissues. The results indicated that HS6ST2 may perform an important role in $\mathrm{GC}$, and the biological mechanism of HS6ST2 requires further exploration.

Heparan sulfate 6-O-sulfotransferase 2 (HS6ST2) is a golgi-resident enzyme that catalyzes the formation of 6-O-sulfation of heparan sulfate (HS) in proteoglycans (HSPGs), which regulate numerous developmental processes $(6,28)$. Following the 6-O-sulfation of HS, HSPGs participate in the regulation of numerous signaling pathways by binding and activating cytokines. Growth factors including epidermal growth factor (EGF), fibroblast growth factor (FGF) and vascular endothelial growth factor (VEGF) are dependent on the 6-O-sulfation of HS to assemble tri-molecular signaling complexes (HS-growth factor-receptor) for signal transduction (29-32). Previous emerging evidence has indicated that HS6ST2 is a critical factor involved in regulating processes of angiogenesis and epithelial-mesenchymal transition (EMT) during carcinogenesis. Through the regulation of HS 6-O-sulfation, HS6ST2 influences angiogenic processes by inducing heparin-binding (HB)-EGF receptor signaling in ovarian cancer (32). EMT is an important mechanism which induces tumor-related epithelial cells to acquire mesenchymal features; including increased motility and reduced cell-cell contact in the early stage of tumorigenesis (33). Song et al (13) revealed that the activation of HS6ST2, in pancreatic cancer (PC), participated in EMT and angiogenesis in the progression of this disease. The potential mechanism by which HS6ST2 potentiates PC carcinogenesis is mainly attributed to the activation of the notch-signaling pathway, which mediates EMT and angiogenesis (13). The notch-signaling pathway is involved in the processes of tumor cell proliferation, invasion, and the establishment of a mesenchymal phenotype $(33,34)$. In thyroid carcinomas, HS6ST2 was identified as a target gene of twist family bhlh transcription factor 1, a critical regulator of EMT $(10,35)$. Inhibition of H6ST2 in tumor cells impairs cell migration, invasion, tubule formation, and may reverse EMT $(10,13,32)$. Notably, previous reports revealed that the specific inhibition of HS6ST2 by a high molecular weight Escherichia coli K5-derived heparin-like polysaccharide (K5-NSOS), prevented tumor progression in a mouse model of breast cancer metastasis (15). These results indicated that K5-NSOS may be a potential anticancer agent in cancer therapy. In addition, a clinical study revealed that the overexpression of HS6ST2 was associated with colorectal cancer (CRC); and may serve as a predictor for poor prognosis in patients with CRC (12). However, the function of HS6ST2 in
GC remains largely unexplored. To the best of our knowledge, the present study is the first investigation into the expression of HS6ST2 in gastric cancer and its clinical significance in the development and progression of this disease.

Gene chip analysis revealed that HS6ST2 was upregulated ( $>12$-fold) in tumor tissues as compared with adjacent non-tumor gastric mucosa. In addition, analysis by RT-qPCR and IHC, confirmed that HS6ST2 mRNA and protein expression levels were significantly higher in tumor tissues. Furthermore, HS6ST2 expression was revealed to be positively associated with TNM stage, invasion depth, and distant metastasis in patients with GC. The prognosis analysis based on IHC and the merged dataset demonstrated that patients with high HS6ST2 expression were associated with a poor prognosis of GC in comparison to those patients with a low expression. Furthermore, multivariate analysis demonstrated that the expression status of HS6ST2 was an independent prognostic factor in patients with GC. It was identified that HS6ST2 is involved in GC as a molecular biomarker, which provides evidence supporting an association between the biological activity of HS6ST2 and GC prognosis. The present study indicates that HS6ST2 may serve as a potential marker for GC and may be useful for the development of effective treatments against GC. In the present study, the results revealed that HS6ST2 was upregulated in GC tissues and may represent a useful prognostic marker. However, the function of HS6ST2 in these processes is largely unclear, and further studies (such as cell biological experiments in vitro and animal experiments in vivo) are needed to characterize the molecular mechanisms by which HS6ST2 participates in the carcinogenesis of GC.

In summary, HS6ST2 was significantly overexpressed in GC tissues, and upregulation of HS6ST2 was associated with aggressive histopathological features and poor prognosis in patients with GC. Taken together, these results indicate that HS6ST2 may be a potential therapeutic target or a valuable prognostic biomarker for patients with GC.

\section{Acknowledgements}

The present study was supported by Zhejiang Provincial Natural Science Foundation of China (grant no. LZ12H16004).

\section{References}

1. Ferlay J, Soerjomataram I, Dikshit R, Eser S, Mathers C, Rebelo M, Parkin DM, Forman D and Bray F: Cancer incidence and mortality worldwide: Sources, methods and major patterns in GLOBOCAN 2012. Int J Cancer 136: E359-E386, 2015.

2. Chen W, Zheng R, Zhang S, Zhao P, Zeng H, Zou X and He J: Annual report on status of cancer in China, 2010. Chin J Cancer Res 26: 48-58, 2014.

3. Allemani C, Weir HK, Carreira H, Harewood R, Spika D, Wang XS, Bannon F, Ahn JV, Johnson CJ, Bonaventure A, et al: Global surveillance of cancer survival 1995-2009: Analysis of individual data for $25,676,887$ patients from 279 population-based registries in 67 countries (CONCORD-2). Lancet 385: 977-1010, 2015

4. Lin LL, Huang HC and Juan HF: Revealing the molecular mechanism of gastric cancer marker annexin A4 in cancer cell proliferation using exon arrays. PLoS One 7: e44615, 2012.

5. Kasaian K and Jones SJ: A new frontier in personalized cancer therapy: Mapping molecular changes. Future Oncol 7: 873-894, 2011. 
6. Habuchi H, Tanaka M, Habuchi O, Yoshida K, Suzuki H, Ban K and Kimata K: The occurrence of three isoforms of heparan sulfate 6-O-sulfotransferase having different specificities for hexuronic acid adjacent to the targeted $\mathrm{N}$-sulfoglucosamine. J Biol Chem 275: 2859-2868, 2000.

7. Habuchi H, Miyake G, Nogami K, Kuroiwa A, Matsuda Y, Kusche-Gullberg M, Habuchi O, Tanaka M and Kimata K: Biosynthesis of heparan sulphate with diverse structures and functions: Two alternatively spliced forms of human heparan sulphate 6-O-sulphotransferase-2 having different expression patterns and properties. Biochem J 371: 131-142, 2003.

8. Nagai N, Habuchi H, Esko JD and Kimata K: Stem domains of heparan sulfate 6-O-sulfotransferase are required for Golgi localization, oligomer formation and enzyme activity. J Cell Sci 117: 3331-3341, 2004.

9. Sasisekharan R, Shriver Z, Venkataraman G and Narayanasami U: Roles of heparan-sulphate glycosaminoglycans in cancer. Nat Rev Cancer 2: 521-528, 2002.

10. Di Maro G, Orlandella FM, Bencivenga TC, Salerno P, Ugolini C, Basolo F, Maestro R and Salvatore G: Identification of targets of Twist1 transcription factor in thyroid cancer cells. J Clin Endocrinol Metab 99: E1617-E1626, 2014.

11. Schulten HJ, Al-Mansouri Z, Baghallab I, Bagatian N, Subhi O, Karim S, Al-Aradati H, Al-Mutawa A, Johary A, Meccawy AA, et al: Comparison of microarray expression profiles between follicular variant of papillary thyroid carcinomas and follicular adenomas of the thyroid. BMC Genomics 16 Suppl 1: S7, 2015

12. Hatabe S, Kimura H, Arao T, Kato H, Hayashi H, Nagai T, Matsumoto K, DE Velasco M, Fujita Y, Yamanouchi G, et al: Overexpression of heparan sulfate 6-sulfotransferase-2 in colorectal cancer. Mol Clin Oncol 1: 845-850, 2013.

13. Song K, Li Q, Peng YB, Li J, Ding K, Chen LJ, Shao CH, Zhang LJ and Li P: Silencing of hHS6ST2 inhibits progression of pancreatic cancer through inhibition of Notch signalling. Biochem J 436: 271-282, 2011.

14. Backen AC, Cole CL, Lau SC, Clamp AR, McVey R, Gallagher JT and Jayson GC: Heparan sulphate synthetic and editing enzymes in ovarian cancer. Br J Cancer 96: 1544-1548, 2007.

15. Pollari S, Käkönen RS, Mohammad KS, Rissanen JP, Halleen JM, Wärri A, Nissinen L, Pihlavisto M, Marjamäki A, Perälä M, et al: Heparin-like polysaccharides reduce osteolytic bone destruction and tumor growth in a mouse model of breast cancer bone metastasis. Mol Cancer Res 10: 597-604, 2012.

16. Waaijer CJ, de Andrea CE, Hamilton A, van Oosterwijk JG Stringer SE and Bovée JV: Cartilage tumour progression is characterized by an increased expression of heparan sulphate 6O-sulphation-modifying enzymes. Virchows Arch 461: 475-481, 2012

17. Edge SB and Compton CC: The American Joint Committee on Cancer: The 7th edition of the AJCC cancer staging manual and the future of TNM. Ann Surg Oncol 17: 1471-1474, 2010.

18. Livak KJ and Schmittgen TD: Analysis of relative gene expression data using real-time quantitative PCR and the 2(-Delta Delta C(T)) method. Methods 25: 402-408, 2001.

19. Györffy B and Schäfer R: Meta-analysis of gene expression profiles related to relapse-free survival in 1,079 breast cancer patients. Breast Cancer Res Treat 118: 433-441, 2009.

20. Barrett T, Wilhite SE, Ledoux P, Evangelista C, Kim IF, Tomashevsky M, Marshall KA, Phillippy KH, Sherman PM, Holko M, et al: NCBI GEO: Archive for functional genomics data sets-update. Nucleic Acids Res 41: D991-D995, 2013.
21. Fekete T, Rásó E, Pete I, Tegze B, Liko I, Munkácsy G, Sipos N, Rigó J Jr and Györffy B: Meta-analysis of gene expression profiles associated with histological classification and survival in 829 ovarian cancer samples. Int J Cancer 131: 95-105, 2012.

22. Mihaly Z, Kormos M, Lánczky A, Dank M, Budczies J, Szász MA and Győrffy B: A meta-analysis of gene expression-based biomarkers predicting outcome after tamoxifen treatment in breast cancer. Breast Cancer Res Treat 140: 219-232, 2013.

23. Orditura M, Galizia G, Sforza V, Gambardella V, Fabozzi A, Laterza MM, Andreozzi F, Ventriglia J, Savastano B, Mabilia A, et al: Treatment of gastric cancer. World J Gastroenterol 20: 1635-1649, 2014.

24. Washington K: 7th edition of the AJCC cancer staging manual: Stomach. Ann Surg Oncol 17: 3077-3079, 2010.

25. Sawada T, Yashiro M, Sentani K, Oue N, Yasui W, Miyazaki K, Kai K, Fushida S, Fujimura T, Ohira M, et al: New molecular staging with G-factor supplements TNM classification in gastric cancer: A multicenter collaborative research by the Japan Society for Gastroenterological Carcinogenesis G-project committee. Gastric Cancer 18: 119-128, 2015.

26. Harris TJ and McCormick F: The molecular pathology of cancer. Nat Rev Clin Oncol 7: 251-265, 2010.

27. D'Angelo G, Di Rienzo T and Ojetti V: Microarray analysis in gastric cancer: A review. World J Gastroenterol 20: 11972-11976, 2014.

28. Bernfield M, Götte M, Park PW, Reizes O, Fitzgerald ML, Lincecum J and Zako M: Functions of cell surface heparan sulfate proteoglycans. Annu Rev Biochem 68: 729-777, 1999.

29. Robinson CJ, Harmer NJ, Goodger SJ, Blundell TL and Gallagher JT: Cooperative dimerization of fibroblast growth factor 1 (FGF1) upon a single heparin saccharide may drive the formation of 2:2:1 FGF1.FGFR2c.heparin ternary complexes. J Biol Chem 280: 42274-42282, 2005.

30. Robinson CJ, Mulloy B, Gallagher JT and Stringer SE: VEGF165-binding sites within heparan sulfate encompass two highly sulfated domains and can be liberated by K5 lyase. J Biol Chem 281: 1731-1740, 2006.

31. Esko JD and Lindahl U: Molecular diversity of heparan sulfate. J Clin Invest 108: 169-173, 2001.

32. Cole CL, Rushton G, Jayson GC and Avizienyte E: Ovarian cancer cell heparan sulfate 6-O-sulfotransferases regulate an angiogenic program induced by heparin-binding epidermal growth factor (EGF)-like growth factor/EGF receptor signaling. J Biol Chem 289: 10488-10501, 2014.

33. De Craene B and Berx G: Regulatory networks defining EMT during cancer initiation and progression. Nat Rev Cancer 13: 97-110, 2013

34. Jie Zou, Peng Li, Fei Lu, Na Liu, Jianjian Dai, Jingjing Ye, Xun Qu, Xiulian Sun, Daoxin Ma, Jino Park and Chunyan Ji: Notch1 is required for hypoxia-induced proliferation, invasion and chemoresistance of T-cell acute lymphoblastic leukemia cells. J Hematol Oncol 6: 3, 2013.

35. Yang J, Mani SA, Donaher JL, Ramaswamy S, Itzykson RA, Come C, Savagner P, Gitelman I, Richardson A and Weinberg RA: Twist, a master regulator of morphogenesis, plays an essential role in tumor metastasis. Cell 117: 927-939, 2004. 